\title{
miR-125a induces apoptosis, metabolism disorder and migration impairment in pancreatic cancer cells by targeting Mfn2-related mitochondrial fission
}

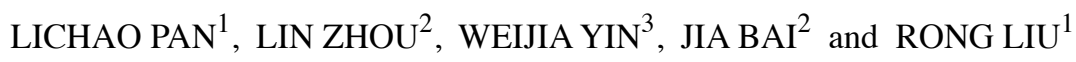 \\ ${ }^{1}$ The Second Department of Hepatobiliary Surgery; Departments of ${ }^{2}$ Hepatobiliary Surgery and \\ ${ }^{3}$ Biochemistry, Chinese PLA General Hospital, Beijing 100853, P.R. China
}

Received December 11, 2017; Accepted April 2, 2018

DOI: $10.3892 /$ ijo.2018.4380

\begin{abstract}
Mitochondrial fission is important for the development and progression of pancreatic cancer (PC). However, little is known regarding its role in pancreatic cancer apoptosis, metabolism and migration. In the current study, the mechanism by which mitochondrial fission modifies the biological characteristics of PC was explored. MicroRNA-125a (miR-125a) had the ability to inhibit mitochondrial fission and contributed to cellular survival. Suppressed mitochondrial fission led to a reduction in mitochondrial debris, preserved the mitochondrial membrane potential, inhibited mitochondrial permeability transition pore opening, ablated cytochrome $c$ leakage into the cytoplasm and reduced the pro-apoptotic protein contents, finally blocking mitochondria related apoptosis pathways. Furthermore, defective mitochondrial fission induced by miR-125a enhanced mitochondria-dependent energy metabolism by promoting activity of electron transport chain complexes. Furthermore, suppressed mitochondrial fission also contributed to PANC-1 cell migration by preserving the F-actin balance. Furthermore, mitofusin 2 (Mfn2), the key defender of mitochondrial fission, is involved in inhibition of miR125a-mediated mitochondrial fission. Low contents of miR-125a upregulated Mfn2 transcription and expression, leading to inactivation of mitochondrial fission. Ultimately, the current study determined that miR-125a and Mfn2 are regulated by hypoxia-inducible factor 1 (HIF1). Knockdown of HIF1 reversed miR-125a expression, and therefore, inhibited Mfn2 expression, leading to activation of mitochondrial fission. Collectively, the present study demonstrated mitochondrial fission as a tumor suppression process that is regulated by the HIF/miR-125a/Mfn2 pathways, acting to restrict PANC-1
\end{abstract}

Correspondence to: Dr Rong Liu, The Second Department of Hepatobiliary Surgery, Chinese PLA General Hospital, 28 Fuxing Road, Beijing 100853, P.R. China

E-mail: liurong301@126.com

Key words: microRNA-125a, PANC-1, mitofusin 2, mitochondrial fission, apoptosis, hypoxia-inducible factor 1 , migration cell survival, energy metabolism and migration, with potential implications for novel approaches for PC therapy.

\section{Introduction}

Pancreatic cancer (PC) is a malignancy with one of the poorest outcomes (1). It is also the fourth most common cause of cancer mortality in the United States (1). Although several risk factors have been identified, diagnostic methods using specific markers to track the occurrence and progression of PC are lacking (2). Considering that the 5-year overall survival of PC remains $<30 \%$, with a median survival of $18-24$ months (3), it is urgent to understand the pathogenesis of PC to aid the identification of markers that are useful for developing innovative diagnostic and therapeutic methods for treating this disease.

MicroRNAs (miRNAs/miRs) are small, noncoding RNAs that are 18-25 nucleotides in length and are involved in the regulation of cancer development and progression in various types of cancer, acting as either oncogenes or tumor suppressor genes. miRNAs regulate gene expression by binding to the 3'-untranslated regions (3'-UTRs) of specific mRNAs, thus controlling mRNA stability and the efficiency of translation (4,5). Growing evidence suggests that miRNAs have an important role in various biological processes, including cell proliferation, apoptosis, and differentiation (6). A number of miRNAs, including miR-132, miR-34, miR-506, and miR-21, have been reported to be associated with PC via microarrays (7). miR-125a is a novel miRNA that is located at chromosome $19 q 13$ (8). miR-125a is frequently downregulated in several types of human cancer, including breast cancer (9), ovarian cancer (10), lung cancer (11) and medulloblastoma (12). Low expression of miR-125a is associated with potential malignant indicators of enhanced gastric cancer, including tumor size and tumor invasion (13). Thus, it is important to establish whether miR-125a is also involved in PC inhibition and, if so, what molecules link miR-125a with cancer mortality.

Mitochondria are central to several cellular physiological processes that range from regulation of bioenergetics to maintenance of the cellular oxidation-reduction (redox) status to the execution of apoptosis $(14,15)$. The mitochondrial network exists along a spectrum of morphologies, from a highly interconnected, elongated network to a highly fragmented, punctate morphology, 
which is considered to be the mitochondrial dynamics (mitochondrial fission and fusion) (16,17). Notably, the critical regulator of mitochondrial dynamics is mitofusin 2 (Mfn2). Lower Mfn2 is associated with excessive mitochondrial fission, an early event that occurs during cancer cell proliferation, apoptosis, metabolism, cell motility and migration via activation of the intrinsic (mitochondrial) apoptotic pathway (18-20). However, a higher Mfn2 concentration has been implicated in cellular survival, chemoresistance and radiotherapy resistance via inhibition of mitochondrial fission $(21,22)$. However, whether miR-125a is able to regulate PC cell death by modifying Mfn2-inhibited mitochondrial fission remains unknown.

In the current study, it was demonstrated that miR-125a is decreased in PANC-1 cells, accompanied by an increase in the contents of $\mathrm{Mfn} 2$. In addition, reintroduction of miR-125a triggered mitochondrial fission in PANC-1 cells via downregulation of Mfn2 transcription and expression. Excessive mitochondrial fission contributes to activation of mitochondria-dependent apoptosis. Furthermore, extensive mitochondrial fission also impairs cellular migration via induction of F-actin degradation. These findings illustrate that miR-125a has a role in mitochondrial fission and is a potential target to slow the development of PC because it modulates cellular apoptosis, energy metabolism and cellular migration.

\section{Materials and methods}

Cell culture. PANC-1 cell line was purchased from National Infrastructure of Cell Line Resource (Beijing, China). PANC-1 cells were cultured in RPMI-1640 medium (Thermo Fisher Scientific, Inc., Waltham, MA, USA) supplemented with $10 \%$ fetal bovine serum (FBS; HyClone; GE Healthcare Life Sciences, Logan, UT, USA), $1 \%$ L-glutamine and $0.5 \%$ gentamycin (Sigma-Aldrich; Merck KGaA, Darmstadt, Germany) at $37^{\circ} \mathrm{C}$ in an atmosphere of $5 \% \mathrm{CO}_{2}$. Hypoxic conditions were induced in hypoxia chamber in a humidified atmosphere with $94 \% \mathrm{~N}_{2}, 5 \% \mathrm{CO}_{2}$ and $1 \% \mathrm{O}_{2}$ for $24 \mathrm{~h}$ (15). To inhibit the mitochondrial fission, mitochondrial division inhibitor 1 (Mdivi1; $10 \mathrm{mM}$; Sigma-Aldrich; Merck KGaA) was used for $12 \mathrm{~h}$ at $37^{\circ} \mathrm{C}$.

Transfection. The miR-125a mimic (agmir-125a), miR-control (scramble miRNA), miR-125a inhibitor (antagomir), small interfering RNA (siRNA) targeting hypoxia-inducible factor 1 (HIF1) and negative control siRNA (siCtrl) were purchased from GenePharma Co., Ltd. (Shanghai, China). The oligonucleotides used in these studies are as follows: Agmir-125a, 5'-CCACAUGAACGCCCAGAGAUU-3'; scramble miRNA, 5'-GAACGGGAGUACAGAGAGAUU-3'; antagomir, 5'-UAA CAAGACCAGAGAGCUGUU-3'; siHIF1, 5'-GAGGAAAAG GGAAAAUCUAUU-3'; siCtrl, 5'-AAUUCUUAAAUUGGG CUGGUU-3'. siRNA $(1 \mu \mathrm{g} / \mathrm{ml})$ and miRNA $(2 \mu \mathrm{g} / \mathrm{ml})$ were added to the media (per $3.5 \times 10^{4}$ cells/well) supplemented with Lipofectamine $^{\circledR} 2000$ (Thermo Fisher Scientific, Inc.) Media containing siRNAs were replaced with Dulbecco's modified Eagle's medium (DMEM) $12 \mathrm{~h}$ after transfection. Transfection was performed for $72 \mathrm{~h}$ and the knockdown of gene expression was assessed by western blot analysis.

Western blotanalysis. To determine the protein levels, $1 \times 10^{6}$ cells were lysed with radioimmunoprecipitation assay (RIPA) buffer (Thermo Fisher Scientific, Inc.) supplemented with phenylmethylsulfonyl fluoride. The protein concentration was analyzed using the bicinchoninic acid protein assay. Protein $(50 \mu \mathrm{g})$ was separated by $10 \%$ SDS-PAGE and then transferred to polyvinylidene difluoride membranes. The membranes were blocked with $5 \%$ nonfat milk for $1 \mathrm{~h}$ at room temperature and then incubated with primary antibodies: Caspase-3 (cat. no. 9662; 1:2,000), caspase-9 (cat. no. 9508; 1:2,000), Bcl-2 (cat. no. $3498 ; 1: 2,000)$, X-linked inhibitor of apoptosis (X-IAP; cat. no. 2042; 1:1,000) and Mfn2 (cat. no. 11925; 1:1,000) from Cell Signaling Technology, Inc. (Danvers, MA, USA); Bcl-2-associated agonist of cell death (Bad; cat. no. ab32445; 1:1,000), Bcl-2 associated $\mathrm{X}$, apoptosis regulator (Bax; cat. no. ab32503; 1:2,000), HIF1 (cat. no. ab16066; 1:1,000), poly(ADP-ribose) polymerase (cat. no. 32064; 1:2,000), complex II (cat. no. ab110410; 1:1,000), complex IV subunit II (cat. no. ab110268; 1:1,000), complex I subunit NDUFB8 (cat. no. ab110242; 1:1,000), density-regulated protein 1 (Drp1; cat. no. ab56788; 1:1,000), mitochondrial fission 1 protein (Fis1; cat. no. ab71498; 1:1,000), G-actin (cat. no. ab123034; 1:1,000) and F-actin (cat. no. ab205; 1:1,000) from Abcam (Cambridge, MA, USA); and complex III subunit core (1:1,000; cat. no. 459220) from Invitrogen (Thermo Fisher Scientific, Inc.) overnight at $4^{\circ} \mathrm{C}$. The membranes were washed in TBS Tween-20 for $15 \mathrm{~min}$ and then incubated with a horseradish peroxidase-conjugated secondary antibody (cat. nos. sc-2004 and sc-2005; Santa Cruz Biotechnology, Inc., Dallas, TX, USA) for $1 \mathrm{~h}$ at room temperature. Blots were detected via an enhanced chemiluminescence substrate kit (Thermo Fisher Scientific, Inc.), and were analyzed using Quantity One 4.6 software (Bio-Rad Laboratories, Inc., Hercules, CA, USA) (23).

Construction of adenovirus for Mfn 2 overexpression. To overexpress Mfn2, the pDC316-mCMV-Mfn2 plasmid (pDC316-mCMV-Mfn2; NheI-forward, 5'-TATCTCATCAGA TTGAGCTCGTCCA-3' and HindIII-reverse, 5'-CGCCTT AGATCCACTCACTGTAGATTCGA-3') was purchased from Vigene Biosciences, Inc. (Rockville, MD, USA). This pDC316-mCMV-Mfn2 plasmid (2.5 $\mu \mathrm{g}$, per $3.5 \times 10^{4}$ cells/well) was transfected into 293T cells (National Infrastructure of Cell Line Resource) in RPMI-1640 medium supplemented with $10 \%$ FBS using Lipofectamine ${ }^{\circledR} 2000$ according to the manufacturer's protocol. The viral supernatant was collected $48 \mathrm{~h}$ after transfection. Supernatant was acquired again and filtered through a $0.45-\mu \mathrm{m}$ filter to obtain the adenovirus-Mfn2 (Ad-Mfn2). A total of $1 \times 10^{5}$ cells/well were infected with 100 multiplicity of infection adenovirus in serum-free DMEM for $6 \mathrm{~h}$ at $37^{\circ} \mathrm{C}$, following which the media was replaced with DMEM supplemented with $10 \%$ FBS.

Reverse transcription-quantitative polymerase chain reaction $(R T-q P C R)$. Total RNA was extracted with TRIzol ${ }^{\circledR}$ reagent (Invitrogen; Thermo Fisher Scientific, Inc.) and reverse transcribed with a One-step RT-PCR kit (TransGen Biotech Co., Ltd., Beijing, China) at $42^{\circ} \mathrm{C}$ for 3 min according to the manufacturer's instructions. The mRNA levels were determined by RT-qPCR in triplicate for each of the independently prepared RNAs and were normalized to the levels of GAPDH expression (24). Gene expression was determined 90 using cBNA SYBR-Green real-time PCR Master Mix (Takara Bio, Inc., 
Otsu, Japan) and qPCR. Primers for qPCR used were as follows: miR-125a reverse transcription primer, 5'-GTCGTATCCAG TGCAGGGTCCGAGGTATTCGCACTGGATACGACTCAC AGG-3'; miR-125a PCR primers, sense, 5'-CTGGAGUCCCUG AGACCCUUUA-3' and antisense, 5'-ACGCTTCACGAATTT GCGTGTC-3'; GAPDH, sense, 5'-TGAGTGCTGTCTCCAT GTTTGA-3' and antisense, 5'-TCTGCTCCCCACCTCTAAG TTG-3'. qPCR was performed at $94^{\circ} \mathrm{C}$ for $3 \mathrm{~min}$ and $94^{\circ} \mathrm{C}$ for $30 \mathrm{sec}$ for $38 \mathrm{cycles}$, and finally $51^{\circ} \mathrm{C}$ for $30 \mathrm{sec}$. The mRNA ratio of the target genes to $\beta$-actin was calculated using the $2^{-\Delta \Delta C q}$ formula (25).

Terminal deoxynucleotidyl transferase dUTP nick end labelling (TUNEL) staining. A TUNEL assay was performed using a one-step TUNEL kit (Beyotime Institute of Biotechnology, Haimen, China) according to the manufacturer's instructions. TUNEL staining was performed with fluorescein-dUTP (Invitrogen; Thermo Fisher Scientific, Inc.) to stain apoptotic cell nuclei, and DAPI $(5 \mathrm{mg} / \mathrm{ml})$ was used to stain all cell nuclei at room temperature for $3 \mathrm{~min}$. Cells in which the nucleus was stained with fluorescein-dUTP were defined as TUNEL positive. The slides were then imaged under a confocal microscope (26).

Hypoxia treatment. Hypoxic conditions were induced using fresh PBS solution with $94 \% \mathrm{~N}_{2}, 1 \% \mathrm{O}_{2}$ and $5 \% \mathrm{CO}_{2}$. The $\mathrm{pH}$ was adjusted to $\mathrm{pH} 6.8$ with lactate (Sigma-Aldrich; Merck KGaA) to mimic ischemic conditions. The dishes were placed into a hypoxia incubator that was equilibrated with $94 \% \mathrm{~N}_{2}, 1 \% \mathrm{O}_{2}$ and $5 \% \mathrm{CO}_{2}$. Deferoxamine (DFX; $10 \mathrm{mg} / \mathrm{ml}$ ) was used to treat cells for $\sim 4 \mathrm{~h}$ to induce the hypoxia condition at $37^{\circ} \mathrm{C}$.

Cell viability assay. Cell proliferation was measured with a Cell Counting Kit-8 (CCK-8) assay (Beyotime Institute of Biotechnology) (27). Briefly, $200 \mu 1$ of the cell suspension was seeded in 96-well cell culture plates at a density of 1,000 cells/well and incubated at $37^{\circ} \mathrm{C}$ for $1-4$ days as previously described (28).

Cell migration assay. Following treatments, PANC-1 cells were seeded at a density of $0.5 \times 10^{6}$ cells/well in 6 -well plates and then cultured overnight ( $90 \%$ confluence). A wound track was scored in each dish with a pipette head. Debris was removed by washing with PBS. After 0,24 and $48 \mathrm{~h}$ of culturing, the migration distances were visualized and imaged (Olympus IX71; Olympus Corporation, Tokyo, Japan). Cell migration was also analyzed using a Transwell chamber assay (24 wells, $8-\mu \mathrm{m}$ pore size with a polycarbonate membrane) as previously described (29).

Determination of caspase-3/9 activity, glucose uptake and lactate production. A caspase-3 activity kit and a caspase- 9 activity kit (Beyotime Institute of Biotechnology) were used to detect the activity of caspase-3 and caspase-9, respectively, according to the manufacturer's protocol (30). Following the appropriate treatments, cultured cells were lysed with RIPA lysis buffer (Beyotime Institute of Biotechnology) for $30 \mathrm{~min}$ and centrifuged at $14,000 \mathrm{xg}$ for $30 \mathrm{~min}$ at $4^{\circ} \mathrm{C}$. The absorbance was measured at a wavelength of $405 \mathrm{~nm}$ using a microplate reader (BioTek Instruments, Inc., Winooski, VT, USA). The data are expressed as the ratio of the optical density (OD) value of the treated group to the OD of the control group. The extracellular lactate was measured using the cell culture medium with lactate assay kit (cat. no. K607-100; BioVision, Inc., Milpitas, CA, USA). Intracellular glucose was measured using cell lysates with glucose assay kit (cat. no. K606-100; BioVision, Inc.). The uptake of glucose, the production of lactate and the levels of ATP were all measured according to the manufacturer's instruction. The assay was repeated three times.

Microchondrial DNA (mtDNA) strand breaks, copy numbers and transcription level detection. mtDNA strand breaks were detected based on methods described previously (11). Briefly, a $200 \mu \mathrm{l}$ cell $\left(1 \times 10^{6}\right)$ suspension was centrifuged at $15,000 \mathrm{x} \mathrm{g}$ at $4^{\circ} \mathrm{C}$ for $20 \mathrm{~min}$. The supernatant was discarded and $400 \mu \mathrm{l} \mathrm{solu}-$ tion $\left(0.25 \mathrm{mmol} / \mathrm{l}\right.$ inositol, $10 \mathrm{mmol} / 1 \mathrm{Na}_{3} \mathrm{PO}_{4}$ and $1 \mathrm{mmol} / \mathrm{l}$ $\mathrm{MgCl}_{2}, \mathrm{pH}$ 7.2) was added at $4^{\circ} \mathrm{C}$ for $30 \mathrm{~min}$ (31). The relative amounts of mtDNA and nuclear DNA content were used to assess the mtDNA copy numbers via qPCR, which was performed as described above. The mtDNA and nuclear amplicons were generated from a complex IV sequence and GAPDH segment, respectively. The mtDNA primers were 5'-CTATGT CGTGTCCAGAG-3' and 5'-CATGTTGTCCCGTGTCATG-3'. The GAPDH primers, chosen as the internal standards, were 5'-CTCAGTCGTATTCGAGTGGTCCT-3' and 5'-CCTGTG GAAGTCCACAACATGTC-3'. The transcript level of mtDNA was reflected by two different components: NADH dehydrogenase subunit 1 (ND1) and cytochrome $c$ oxidase subunit I. The primers for cytochrome $c$ oxidase subunit I were 5'-ATCGTT CGGTGAGGTCGTG-3' and 5'-CGCCGGTGTCATTATCGT ATA-3'. The primers for ND1 were 5'-TTGCCGTATATTCAGT ATC-3' and 5'-ATCCTGTTGCCCAGTCCAGT-3'. GAPDH was selected as the internal standard (32).

ATP production, JC-1 staining, mitochondrial permeability transition pore (mPTP) opening and mitochondrial respiratory function. The cellular ATP levels were measured using a firefly luciferase-based ATP assay kit (Beyotime Institute of Biotechnology). The opening of mPTP was visualized as a rapid dissipation of tetramethylrhodamine ethyl ester fluorescence as described in a previous study (33). Mitochondrial respiration was initiated by adding glutamate/malate to final concentrations of 5 and $2.5 \mathrm{mmol} / \mathrm{l}$ for $5 \mathrm{~min}$, respectively. State 3 respiration was initiated by adding ADP $(150 \mathrm{nmol} / \mathrm{l})$ for $5 \mathrm{~min}$; state 4 was measured as the rate of oxygen consumption after ADP phosphorylation. The respiratory control ratio (state 3/state 4) and ADP/O ratio (number of nmol ADP phosphorylated to atoms of oxygen consumed) were calculated as previously described (34). Mitochondrial depolarization was evaluated using MitoProbe ${ }^{\mathrm{TM}}$ JC-1 assay kit (Thermo Fisher Scientific Inc.), according to the manufacturer's protocol.

Immunofluorescence staining. To determine cytochrome $c$ (cyt-c) localization and mitochondrial division, immunofluorescence staining was used. Cells were fixed in $3.7 \%$ paraformaldehyde for $10 \mathrm{~min}$ at room temperature and permeabilized in $100 \%$ pre-chilled acetone (Sinopharm Chemical Reagent Co., Ltd., Shanghai, China). Following blocking with 
A

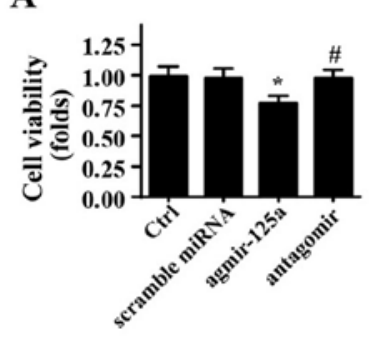

C
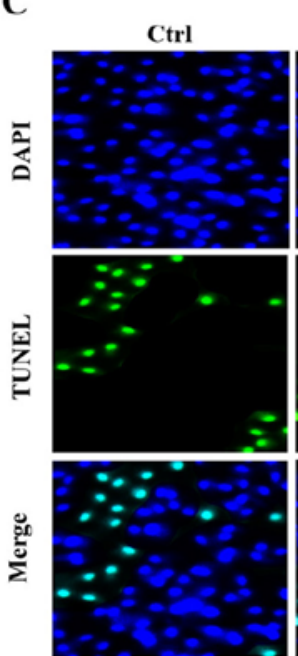

B

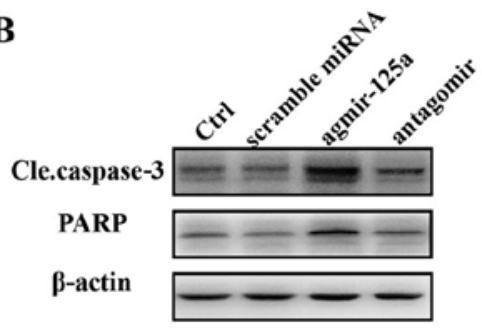

miR-125a mimics
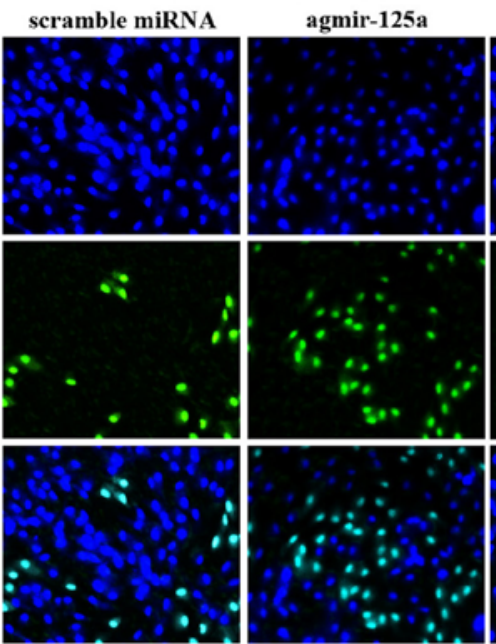
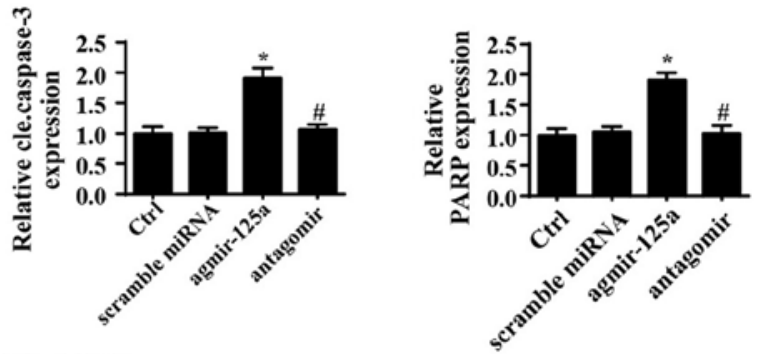

miR-125a inhibitor antagomir

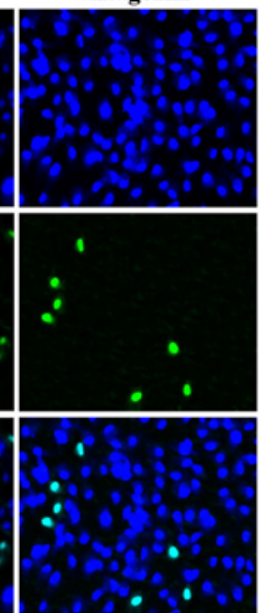

Figure 1. miR-125a is associated with PANC-1 cell survival. (A) CCK-8 assay to evaluate effect of miR-125a on the growth capacity of PANC-1. (B) Cle.caspase-3 and PARP were measured by western blot analysis. (C) TUNEL staining (magnification, $\mathrm{x} 40$ ). ${ }^{*} \mathrm{P}<0.05$ vs. Ctrl; ${ }^{\#} \mathrm{P}<0.05$ vs. agmir-125a. Ctrl, control; miR, microRNA; agmir-125a, miR-125a mimic; antagomir, miR-125a inhibitor; Cle.caspase-3, cleaved caspase-3; PARP, poly(ADP-ribose) polymerase; TUNEL, terminal deoxynucleotidyl transferase dUTP nick end labelling.

$5 \%$ bovine serum albumin (Sigma-Aldrich; Merck KGaA) in PBS for $1 \mathrm{~h}$ at room temperature, the cells were incubated with primary antibodies for $4 \mathrm{~h}$ at room temperature. Subsequently, the cells were incubated with Alexa-Fluor 116488 donkey anti-rabbit secondary antibody $(1: 1,000$; cat. no. A-21206; Invitrogen; Thermo Fisher Scientific, Inc.) at $37^{\circ} \mathrm{C}$ for $1 \mathrm{~h}$ in the dark. Images were captured using a laser confocal microscope (TcS SP5; Leica Microsystems, Inc., Buffalo Grove, IL, USA). The primary antibodies used for cell immunofluorescence were cyt-c (1:500; cat. no. ab90529), translocase of outer mitochondrial membrane 20 (1:500; cat. no. ab56783) and F-actin (1:500; cat. no. ab205) from Abcam. DAPI (5 mg/ml; Sigma-Aldrich; Merck KGaA) was used to stain the nucleus at room temperature for $3 \mathrm{~min}(35)$.

Transmission electron microscopy. Following treatment, cells were collected and fixed with $3 \%$ glutaraldehyde in $100 \mathrm{mM}$ cacodylate buffer at $4^{\circ} \mathrm{C}$ overnight, post-fixed in $1 \%$ cacodylate-buffer osmium tetroxide for $2 \mathrm{~h}$ at room temperature, and dehydrated in a graded series of ethanol (50, 70, 90 and 100\% for $20 \mathrm{~min}$ each). Then, cells were embedded in EponAradite. Ultrathin sections $(60 \mathrm{~nm})$ were cut with a diamond knife on a Leica EM UC6rt (Leica Microsystems GmbH, Wetzlar, Germany) and double-stained with uranyl acetate and lead citrate. The ultrastructure of cells was observed with a Hitachi H7650 transmission electron microscope (TEM; Hitachi, Ltd. Tokyo, Japan) at $80 \mathrm{kV}$. Three slides were used in each experiments and the TEM assay was repeated three times (36).
Luciferase activity assay. Wild-type Mfn2 3'-UTR (WT) and mutant Mfn2 3'-UTR (MUT) containing the putative binding site of miR-125a were chemically synthesized and cloned downstream of the firefly luciferase gene in a pGL3-promoter vector (Promega Corporation, Madison, WI, USA). PANC-1 cells were placed on a 48 -well plate and cultures until $80 \%$ confluence (37). Cells were then co-transfected with luciferase plasmids $\left(2.5 \mu \mathrm{g}\right.$ per $3.5 \times 10^{4}$ cells/well $)$ and miR-125a or control miRNA in DMEM medium supplemented with $10 \%$ FBS using Lipofectamine ${ }^{\circledR} 2000$ according to the manufacturer's protocol. The pRL Renilla control reporter vectors (Promega Corporation) was used as an internal control to normalize the values of the experimental reporter gene. At $48 \mathrm{~h}$ after transfection, the intensities were measured with a Luciferase Reporter Assay System (Promega Corporation).

Mitochondrial reactive oxygen species (Mito-ROS) detection via flow cytometry and electron transport chain complexes (ETCX) activity detection. Mito-ROS levels were measured using flow cytometry with a MitoSOX red mitochondrial superoxide indicator (Molecular Probes; Thermo Fisher Scientific, Inc.). Cells $\left(3.5 \times 10^{6}\right.$ cells/well $)$ were plated in 6-well culture plates. Subsequently, cells were incubated with MitoSOX $(25 \mu \mathrm{M})$ in PBS at $37^{\circ} \mathrm{C}$ for $30 \mathrm{~min}$, washed twice with PBS, and detached by treatment with trypsin-EDTA. The detached cells were collected and resuspended in PBS, and the fluorescence intensity of cells $\left(3.5 \times 10^{6}\right.$ cells/well $)$ was measured using flow cytometry (BD FAcSVerse) and analyzed 
A
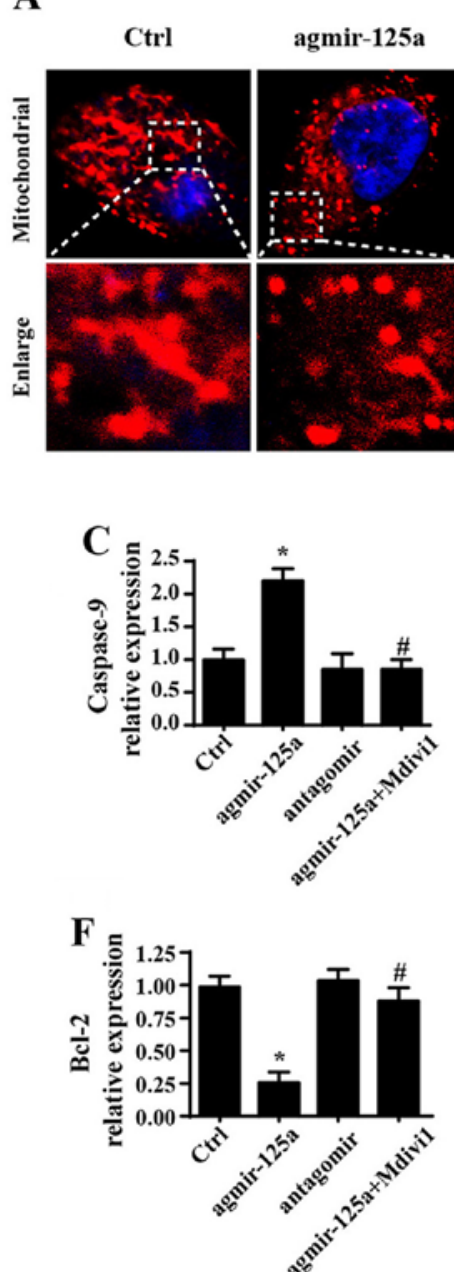

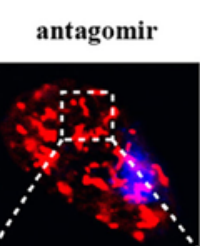

agmir-125a + Mdivi1
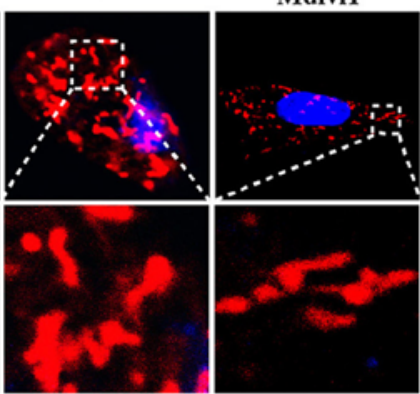

D

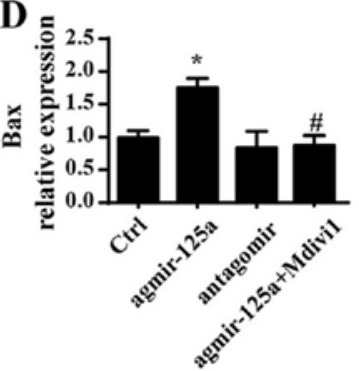

$\mathbf{G}$

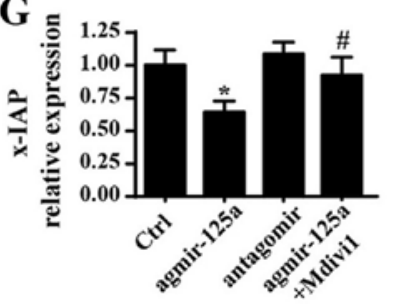

B

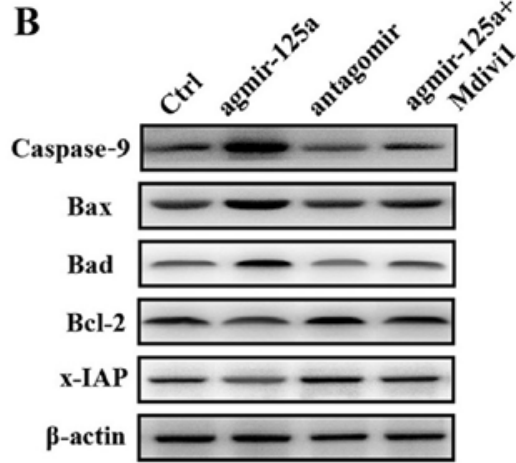

E

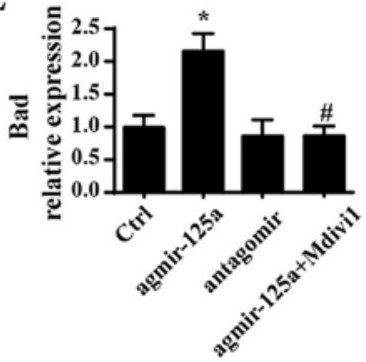

$\mathbf{H}$

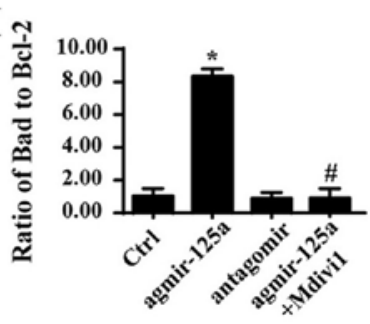

Figure 2. Overexpression of miR-125a promotes cell death by inducing mitochondrial fission-related mitochondrial apoptosis pathways. (A) Change of the mitochondrial morphology via Tomm20 staining. (B) Western blot analysis was performed and densitometry performed for expression of (C) caspase-9, (D) Bax, (E) Bad, (F) Bcl-2 and (G) X-IAP, and (H) Bax/Bcl-2 ratio was calculated.

via BD Paint-A-Gate ${ }^{\mathrm{TM}}$ Pro software (version 4.2) (both from BD Biosciences) (38). ETCx activities were analyzed via ELISA according to the manufacturer's protocol. The ELISA assay kits for ETCX I, II, and V were purchased from Beyotime Institute of Biotechnology (cat. nos. S0052, S0101 and S0052) (39).

Statistical analysis. All analyses were performed with SPSS 20.0 software (IBM Corporation, Armonk, NY, USA). All experiments were repeated three times. The data are presented as the mean \pm standard deviation and statistical significance for each variable was estimated by a one-way analysis of variance followed by Tukey's test for the post hoc analysis. $\mathrm{P}<0.05$ was considered to indicate a statistically significant difference.

\section{Results}

miR-125a enhances PANC-1 cell death. Initially, to verify the role of miR-125a in regulation of the physiological processes of PANC-1 cells, a mimic (agmir-125a) and inhibitor (antagomir) of miR-125a were used. A CCK-8 assay was used to evaluate the growth capacity of PANC-1 cells. As shown in Fig. 1A, agmir-125a reduced the viability of PANC-1 cells, whereas application of antagomir marginally promoted cellular growth, as evidenced by higher OD values in the CCK-8 assay. Furthermore, expression of cleaved caspase- 3 and its substrate, indicators of cellular apoptosis, were also significantly elevated in response to agmir-125a treatment in PANC-1 cells (Fig. 1B). To further understand whether miR-125a modifies cellular survival, TUNEL staining was used (Fig. 1C). Similarly, introduction of miR-125a via agmir-125a augmented the ratio of TUNEL positive cells. By contrast, inhibition of miR-125a reduced the percentage of TUNEL positive cells. These data indicated that miR-125a is a pro-apoptotic factor in PANC-1 cells.

Overexpression of miR-125a promotes cell mitochondrial death by inducing mitochondrial fission. To explore the mechanism by which miR-125a regulates PANC-1 cell death, mitochondrial damage was investigated. Recent studies have suggested that mitochondrial fission is an early event that triggers mitochondria-related apoptosis pathways $(40,41)$. Therefore, the change of mitochondrial morphology was evaluated. As shown in Fig. 2A, compared to spindle mitochondria in the control group, agmir-125a markedly increased the amount of fragmented mitochondria, as evidenced by more round and mitochondrial debris. However, application of the mitochondrial fission inhibitor Mdivi1 blocked the effects 
I
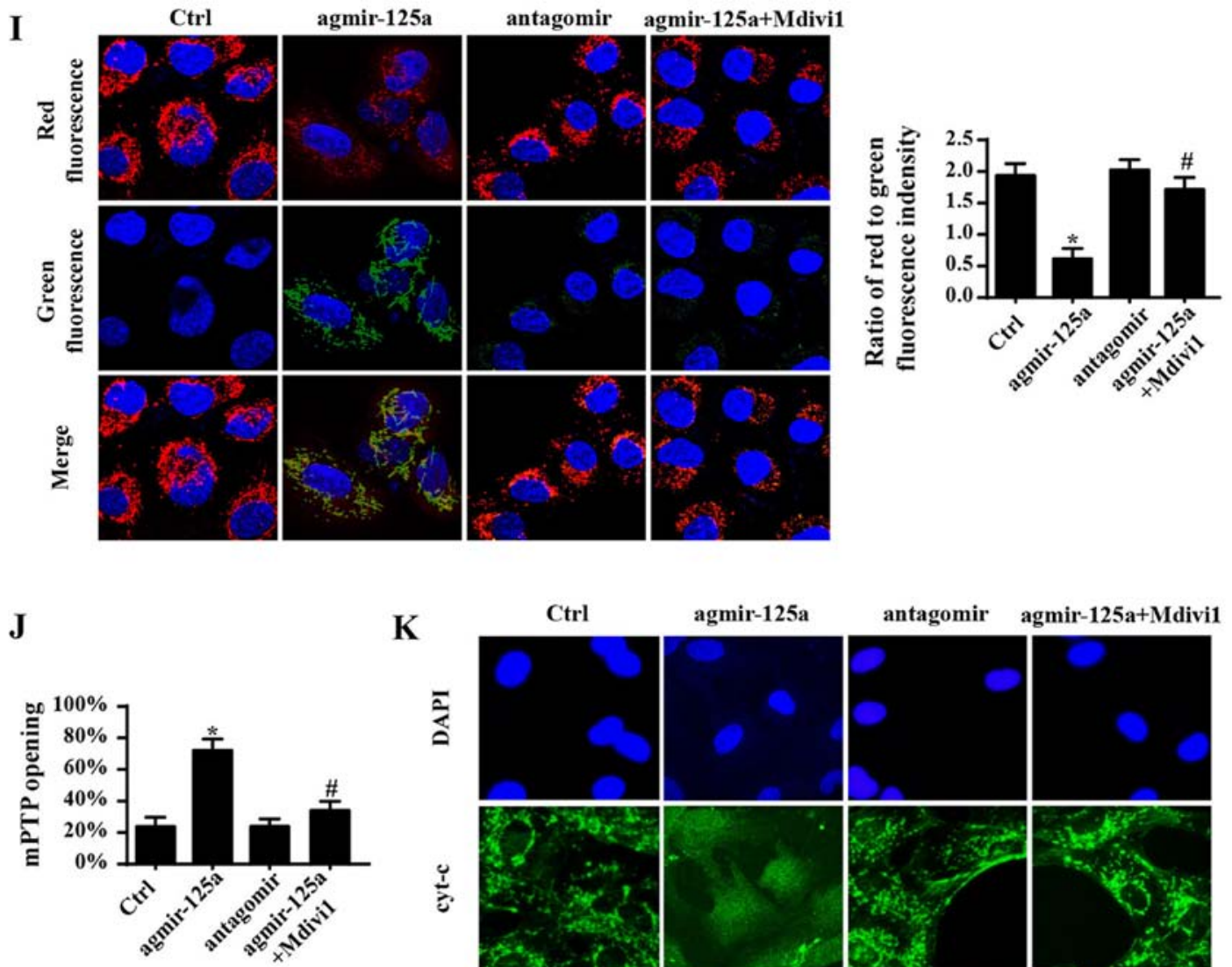

$\mathbf{K}$

Ctrl

agmir-125a

antagomir agmir-125a+Mdivi1
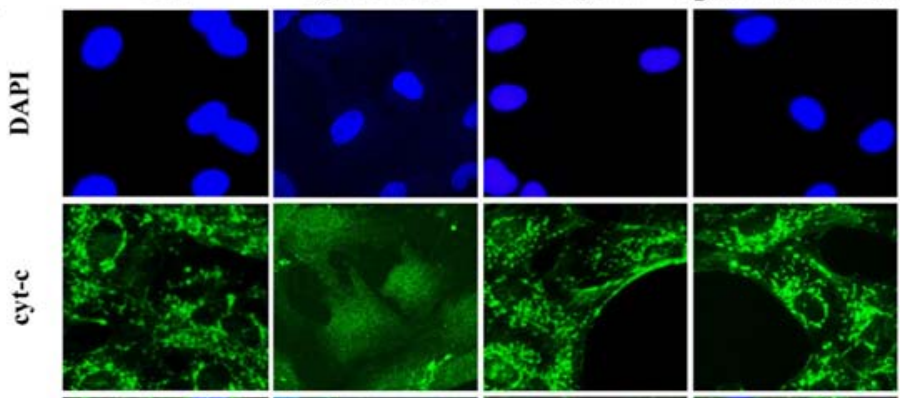

ํํํ
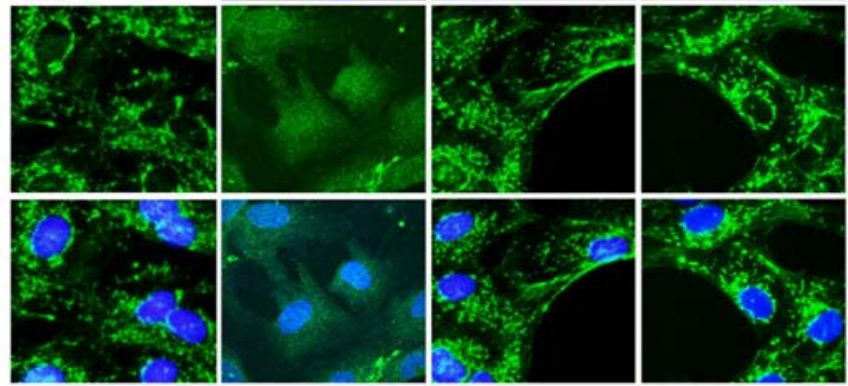

Figure 2. Continued. (I) Mitochondrial membrane potential was measured by JC-1. (J) mPTP opening rate increased upon application of agmir-125a, but decreased when treated with fission inhibitors. (K) cyt-c immunofluorescence showed cyt-c leakage from mitochondria into the cytoplasm induced by agmir-125a, and some cyt-c even migrated into the nucleus. ${ }^{*} \mathrm{P}<0.05$ vs. Ctrl group; ${ }^{*} \mathrm{P}<0.05$ vs. agmir-125a group. Tomm 20 , translocase of outer mitochondrial membrane 20; Ctrl, control; miR, microRNA; agmir-125a, miR-125a mimic; antagomir, miR-125a inhibitor; Mdivi-1, mitochondrial division inhibitor 1; Bax, Bcl-2 associated X, apoptosis regulator; Bad, Bcl-2-associated agonist of cell death; $\mathrm{x}$-IAP, X-linked inhibitor of apoptosis; mPTP, mitochondrial permeability transition pore; cyt-c, cytochrome c.

of agmir-125a on mitochondria fragmentation. By contrast, antagomir treatment led to more mitochondria with a longer length compared with the control group. These data indicated that miR-125a activates mitochondrial fission. Additionally, to investigate whether mitochondrial fission was associated with apoptosis, proteins associated with mitochondrial damage were evaluated. Introduction of agmir-125a increased Bax, Bad and caspase- 9 expression, and reduced $\mathrm{Bcl}-2$ and $\mathrm{x}$-IAP expression, suggesting activation of mitochondria-associated apoptosis pathways (Fig. 2B-H). However, the mitochondrial fission inhibitor blocked the pro-apoptotic effects of agmir-125a. These data indicated that the excessive fission activated by miR-125a contributes to the initiation of mitochondria-associated apoptosis pathways. Furthermore, mitochondrial apoptosis is induced due to mitochondrial membrane potential dissipation, mPTP opening and subsequent cyt-c leakage into the cytoplasm, which activates caspase-9 and caspase-3. Therefore, we observed upstream changes in the context of miR-125a-mediated fission. As shown in Fig. 2I, agmir-125a treatment destroyed the membrane potential with evidence of decreased red fluorescence, but increased green fluorescence.
However, the mitochondrial fission inhibitor reversed these changes. Furthermore, the mPTP opening rate was increased upon application of agmir-125a, but decreased when treated with fission inhibitors (Fig. 2J). The agmir-125a also caused more cyt-c leakage from mitochondria into the cytoplasm, and some cyt-c even migrated into the nucleus (Fig. 2K). However, these changes were blocked by the mitochondrial fission inhibitor. Together, these data indicate that miR-125a activates mitochondrial fission, which induces mitochondrial potential collapse, mPTP opening and cyt-c leakage into the cytoplasm and nucleus, leading to activation of caspase-9-dependent mitochondria apoptosis pathways.

Mitochondrial fission causes mitochondrial energy disorder. In addition to activating apoptosis, the central role of mitochondria is to generate energy and regulate metabolism, which are fundamental for tumor development and progression $(42,43)$. Therefore, we observed energy or metabolism alterations upon miR-125a stimulation. As shown in Fig. 3A, agmir-125a reduced the contents of double-stranded mtDNA (Fig. 3A), mtDNA copy number (Fig. 3B), mtDNA transcripts (Fig. 3C) and ATP 

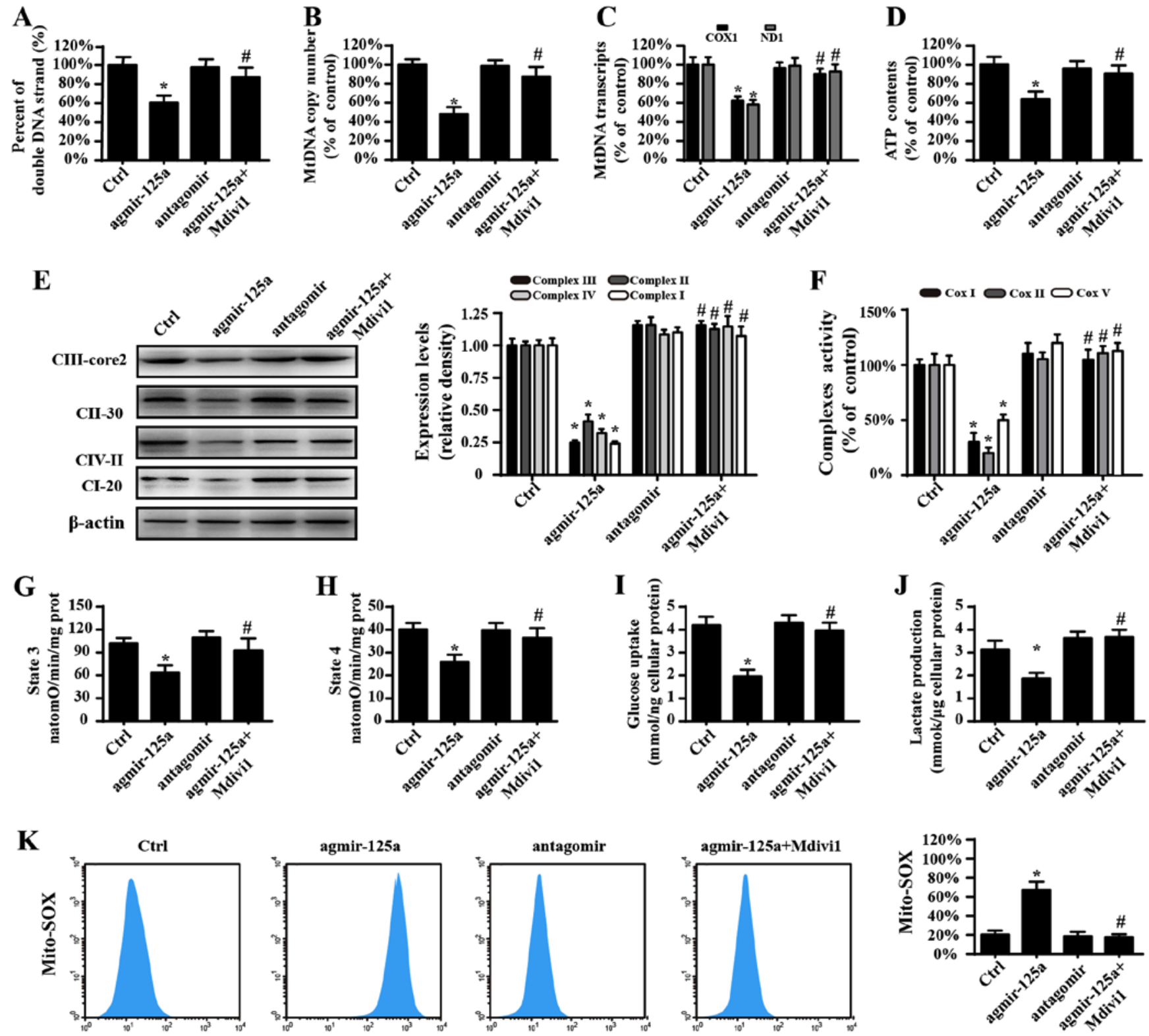

Figure 3. Mitochondrial fission causes mitochondrial energy disorder. (A) The percentage of double-stranded mtDNA indicates mtDNA strand breaks. (B) The mtDNA copy number was assessed by complex IV segments. (C) The transcript levels of mtDNA are reflected by two different components: ND1, encoded by the light chain of mtDNA, and COX I, encoded by the heavy chain of mtDNA. (D) Change in ATP contents. (E) Expression of mitochondrial ETCxs. (F) Changes in the ETCx I, II and V activities as measured via ELISA. Effects of agmir-125a on (G) state 3 respiration, (H) state 4 respiration, respiratory control ratio (RCR [state 3/4]). (I) Glucose uptake. (J) Lactate production. (K) Mito-ROS contents. The curve chart indicates the quantitative flow cytometry results. "P<0.05 vs. Ctrl group; "P<0.05 vs. agmir-125a group. Ctrl, control; miR, microRNA; agmir-125a, miR-125a mimic; antagomir, miR-125a inhibitor; Mdivi-1, mitochondrial division inhibitor 1; COX 1, cytochrome $c$ oxidase subunit I; ND1, NADH dehydrogenase subunit 1; ETCxs, electron transport chain complexes; Mito-ROS, mitochondrial reactive oxygen species.

generation (Fig. 3D) in PANC-1 cells, which were blocked by the fission inhibitor. Electron transport chain complexes (ETCx) are mainly encoded by mtDNA and primarily responsible for ATP generation via using hydrion, electron and oxygen. ETCx expression and activity is vital to delivery hydrion and electron to oxygen, favoring to the mitochondrial oxidative phosphorylation. Accordingly, the downregulation and inactivation of ETCx would predispose the energy undersupply. Based on this, the influence of miR-125a on ETCx expression and activity was evaluated. The results shown in Fig. 3E-J demonstrate that agmir-125a treatment significantly reduced the ETCx concentration and activity, which are coupled to the decrease of the state 3 respiratory rate. Furthermore, agmir-125a-treated
PANC-1 cells took up less glucose and therefore produced less lactate. However, the fission inhibitor abolished the inhibitory effects of agmir-125a on PANC-1 cells. Additionally, the inhibitor of miR-125a slightly elevated ETCx activity $(\mathrm{P}>0.05)$ and the subsequent mitochondrial respiratory function, which were also associated with increased glucose consumption and lactate production. Similar results were observed with mitochondrial reactive oxygen species (Fig. 3K). These data suggest that mitochondrial fission activated by miR-125a impairs PANC-1 cell energy metabolism.

Excessive fission impaired the cellular migration capacity by inducing F-actin depolymerization into G-actin. Apart from 
A

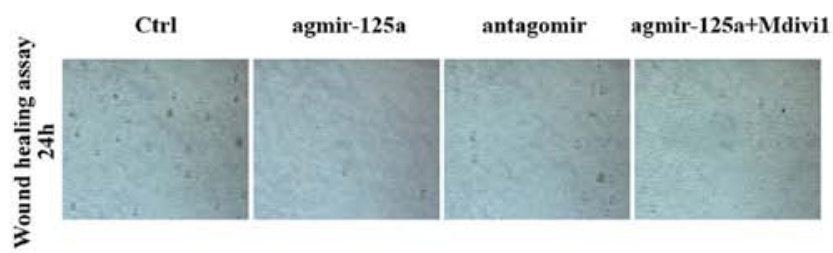

B

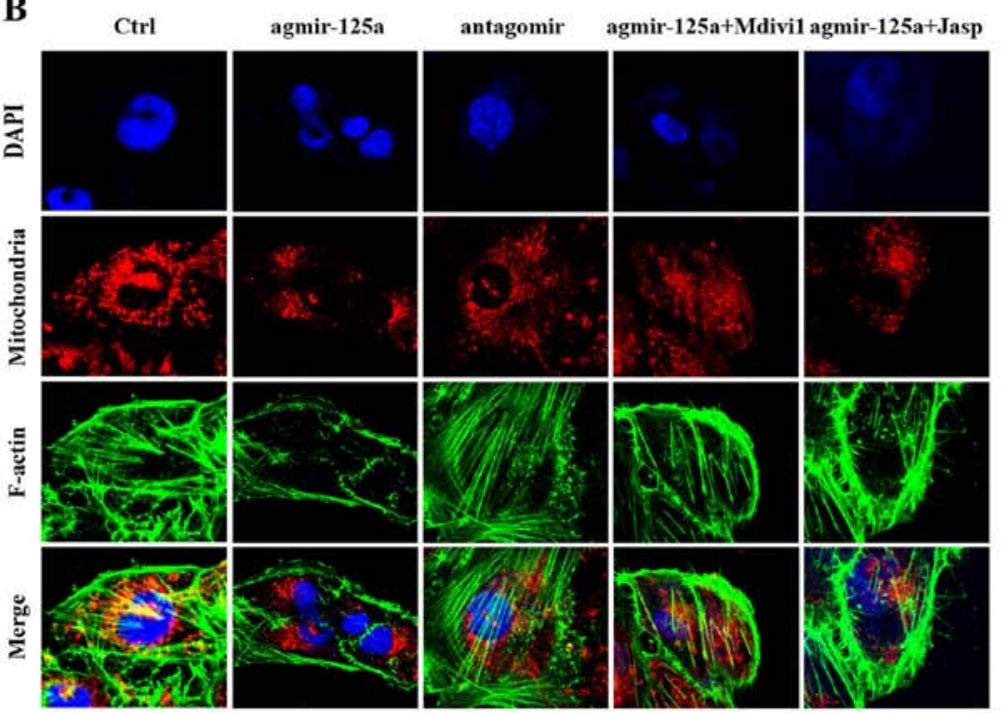

D
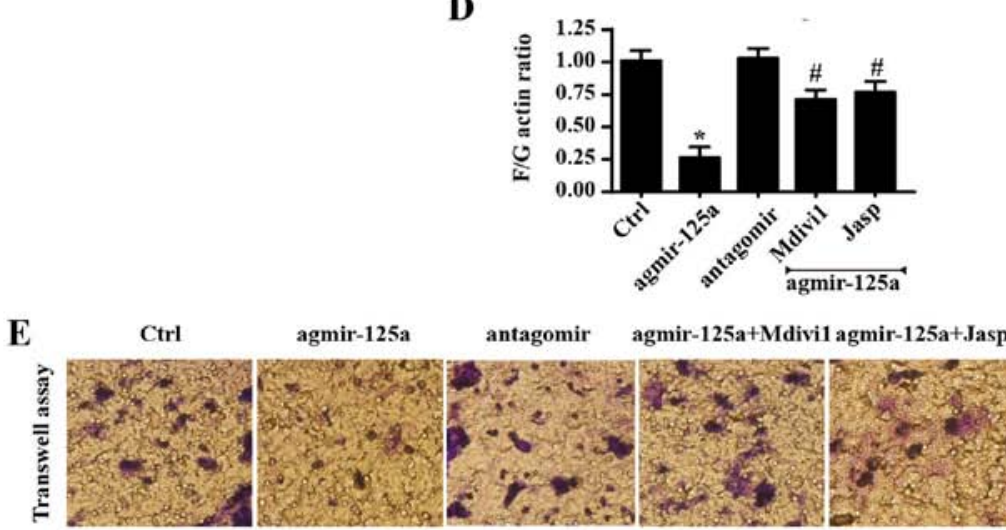

Relative migration distance

(wound healing assay)
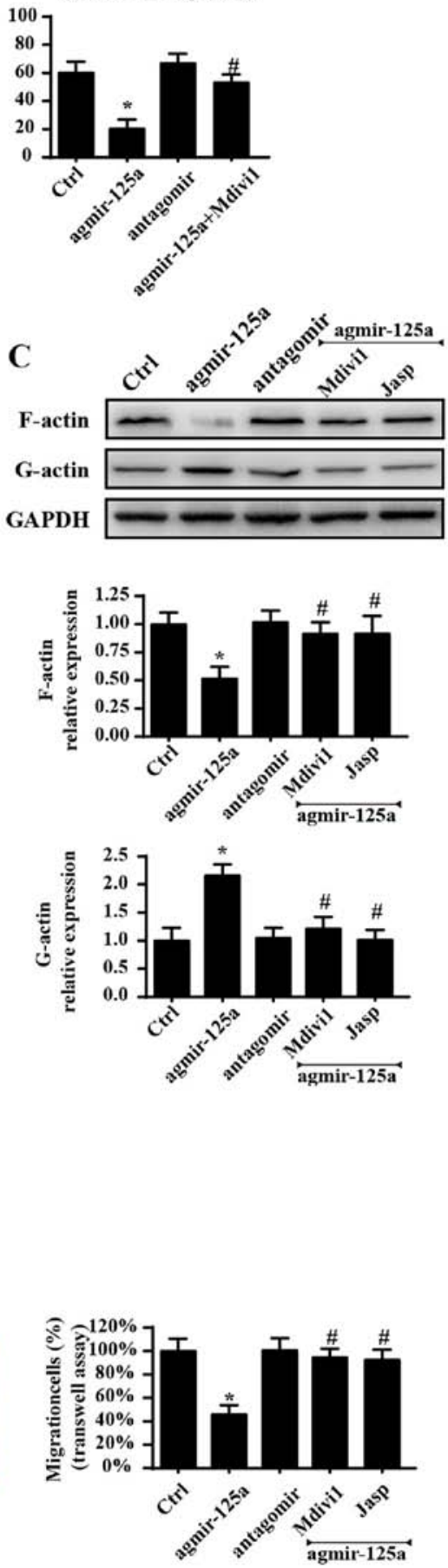

Figure 4. Excessive fission impairs the cellular migration capacity via induction F-actin depolymerization into G-actin. (A) Wound healing assay was used to evaluate the influence of miR-125a on the mobilization capacity of PANC-1 cells. (B) Fluorescence microscopy to obverse the F-actin change; mitochondria stained with Tomm20 antibody and F-actin with rhodamine-phalloidin. (C) Expression of G-actin and F-actin were measured by western blot analysis and (D) the ratio of $\mathrm{F}$-actin to $\mathrm{G}$-actin was determined. (E) Cellular migration capacity was measured by Transwell assay. ${ }^{*} \mathrm{P}<0.05$ vs. Ctrl group; ${ }^{*} \mathrm{P}<0.05$ vs. agmir-125a group. Ctrl, control; miR, microRNA; agmir-125a, miR-125a mimic; antagomir, miR-125a inhibitor; Mdivi-1, mitochondrial division inhibitor 1; Jasp, jasplakinolide.

cellular survival and energy metabolism, cellular migration is another factor that contributes to tumorigenesis (44). Therefore, whether mitochondrial fission has a role in cellular migration was investigated. Initially, a wound-healing assay was used to evaluate the influence of miR-125a on the mobilization of PANC-1 cells. As shown Fig. 4A, exogenously administered agmir-125a reduced the migration of PANC-1 cells, while the mitochondrial fission inhibitor blocked such changes. These data indicated that miR-125a has a role in cellular migration via fission. As F-actin is the key stress fiber that directly regulates cellular mobilization, it was hypothesized that the blunted migration was derived from mitochondrial fission-involved F-actin dyshomeostasis. Fluorescence was also used to obverse F-actin changes. As shown in Fig. 4B, agmir-125a significantly reduced the fluorescence intensity of F-actin, but increased the amount of mitochondrial debris, in PANC-1 cells, which 


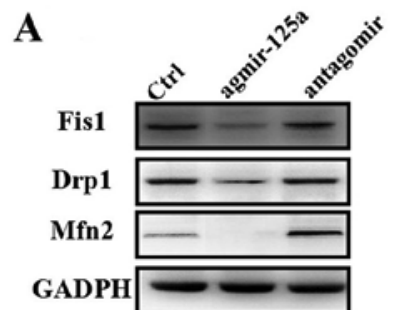

B

Position 126-132 of Mfn2 3'-UTR: 5' ...GUGGUAAUGACCUGUCUCAGGGC... Rno-miR-125a-5p: 3' AGUGUCCAAUUUCCCAGAGUCCCC... Position 126-132 of Mfn2 3'-UTR: 5' ...GUGGUAAUGACCUGUCUCAGGGC... Hsa-miR-125a-5p: 3' AGUGUCCAAUUUCCCAGAgUCCCC.. Position 126-132 of Mfn2 3'-UTR: 5' ...GUGGUAAUGACCUGUCUCAGGGC... Mmu-miR-125a-5p: 3' AGUGUCCAAUUUCCCAGAGUCCCC...

E
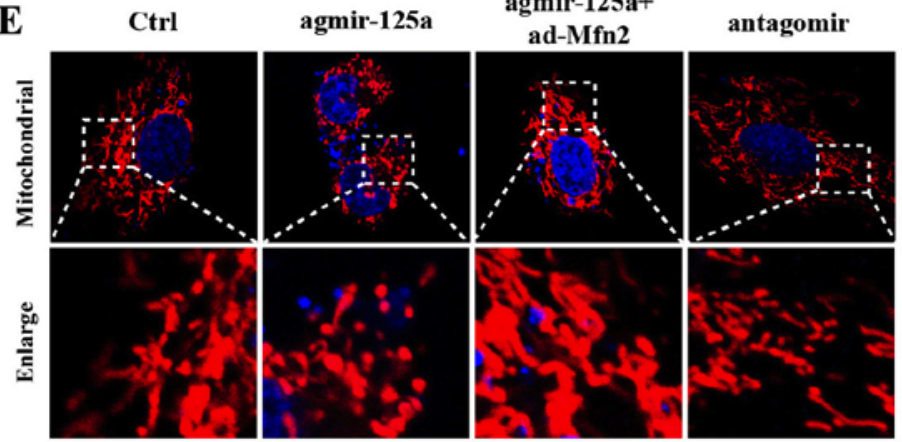
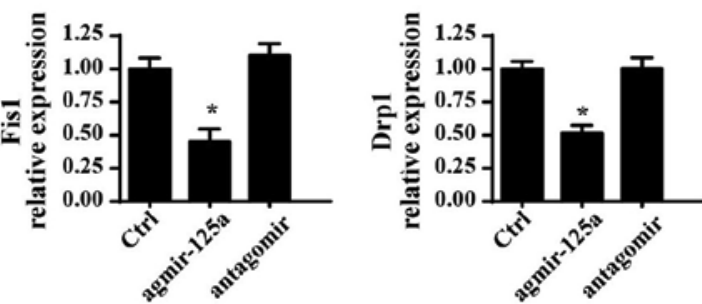

C

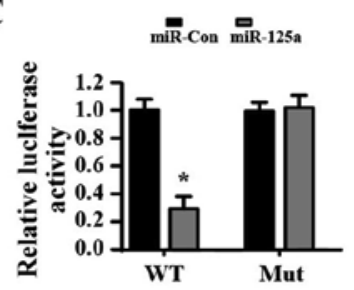

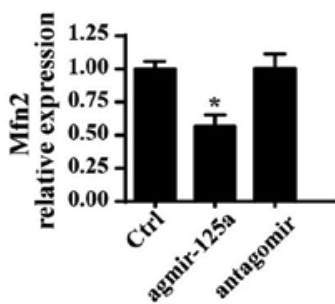

D
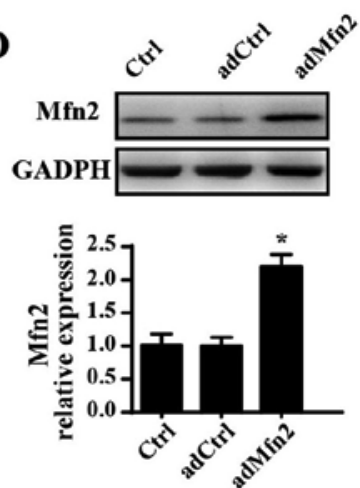

F

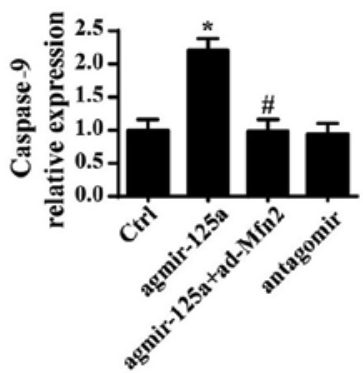

Figure 5. miR-125a activates mitochondrial fission by negatively regulating Mfn2. (A) miR-125a antagomir reversed expression of Fis1, Drp1 and Mfn2 in PANC-1 cells. (B) Computational analysis was performed for miRNA seed sequences complementary to the 3'-UTR of Mfn2 mRNA, which were conserved at the putative binding sites in rat, human, and mouse. (C) Luciferase assay for post-transcriptional repression of Mfn2. (D) The transfection efficiency of Mfn2 evaluated by western blot analysis. (E) The change of mitochondrial fragmentation or debris measured via Tomm20 immunofluorescence after overexpression of Mfn2 via ad-Mfn2. (F) The change of caspase-9 activity following overexpression of Mfn2 via ad-Mfn2. * $\mathrm{P}<0.05$ vs. Ctrl group; ${ }^{\#} \mathrm{P}<0.05$ vs. agmir- $125 \mathrm{a}$ group. Tomm20, translocase of outer mitochondrial membrane 20; Ctrl, control; miR, microRNA; agmir-125a, miR-125a mimic; antagomir, miR-125a inhibitor; Fis1, mitochondrial fission 1 protein; Drp1, density-regulated protein; Mfn2, mitofusin 2; ad-, adenovirus; 3'-UTR, 3'-untranslated region.

were reversed by the mitochondrial fission inhibitor and F-actin depolymerization inhibitors. As F-actin is composed of G-actin, it was hypothesized that there was an imbalance between F-actin and G-actin. As expected, agmir-125a treatment accelerated F-actin division into G-actin, as evidenced by increased $\mathrm{G}$-actin and decreased $\mathrm{F}$-actin protein expression in the agmir-125a group (Fig. 4C and D). Mdivil blocked these conformation alterations, suggesting that mitochondrial fission is responsible for F-actin depolymerization to G-actin. Furthermore, inhibition of F-actin degradation via Jasplakinolide reversed migration under agmir-125a treatment, which is similar to the results in the Mdivil group (Fig. 4A-D). Similar results were observed in Transwell assays (Fig. 4E). Together, these data indicated that miR-125a impairs cellular migration by inducing F-actin depolymerization into G-actin by activating mitochondrial fission.

miR-125a activates mitochondrial fission via negative regulation of Mfn2. To determine the mechanism by which miR-125 regulates mitochondrial fission, the change of $\mathrm{Mfn} 2$, which prevents excessive mitochondrial fission was investigation.
Treatment with agmir-125a reduced the contents of Mfn2 in PANC-1 cells. However, introduction of miR-125a antagomir reversed expression of Mfn2 in PANC-1 cells (Fig. 5A). The similar results were observed for other mitochondrial fission makers, including Fis1 and Drp1. These data indicated that miR-125a negatively regulated the expression of Mfn2. Furthermore, through analysis of the gene sequences, miR-125a was matched to Mfn2, which is highly conserved among rat, human and mouse (Fig. 5B). To test whether miR-125a directly targeted the 3'-UTR of Mfn2, luciferase assays were performed using 3'-UTR sequence fragments containing the predicted target of Mfn2 and its mutated version inserted downstream of a luciferase reporter (Fig. 5C). The results demonstrated that the luciferase activity was downregulated in cells co-transfected with miR-125a mimics and the wild-type Mfn2 3'-UTR compared with the mutated type, suggesting that Mfn2 is a target gene of miR-125a. These data indicate that miR-125a directly regulates transcription and expression of Mfn2.

To confirm whether Mfn2 was responsible for fission, Mfn2 was overexpressed using an adenovirus (Ad-Mfn2) in 

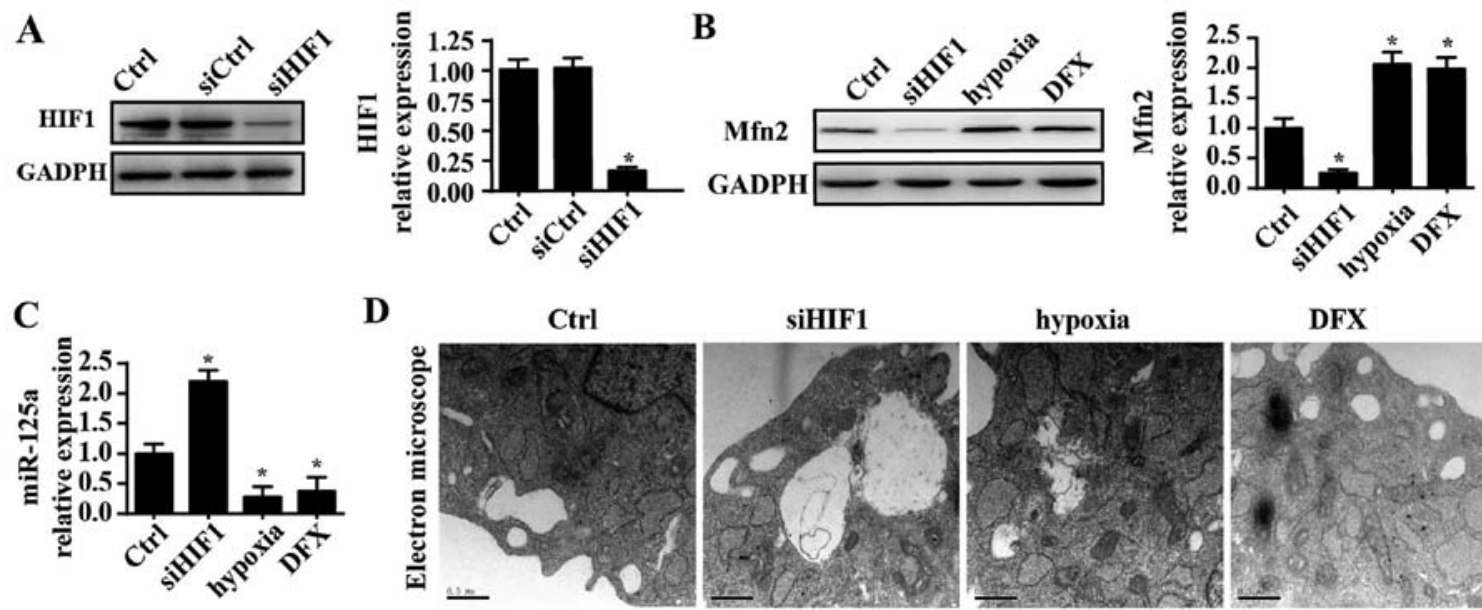

siHIF1

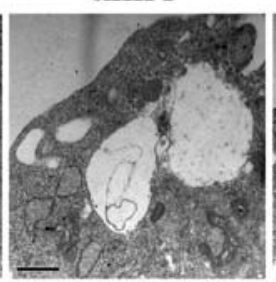

hypoxia

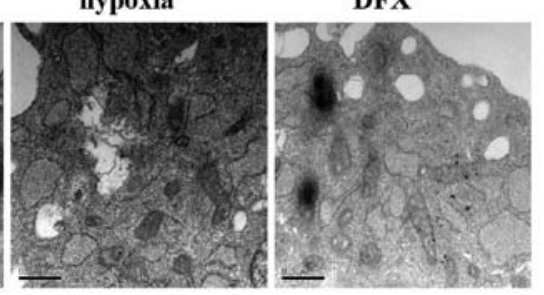

Figure 6. miR-125a is negatively regulated by HIF1. (A) The silencing efficiency of siHIF1 was measured by western blot analysis. (B) The influence of HIF1 on Mfn2 expression was measured by western blot analysis. (C) Knockdown of HIF1 also reduced transcription of Mfn2. (D) Transmission electron microscopy analysis indicated that more fragmented mitochondria appeared in response to HIF1 silencing. "P $<0.05$ vs. Ctrl group. Ctrl, control; si, small interfering RNA; HIF1, hypoxia-inducible factor 1; Mfn2, mitofusin 2; DFX, desferrioxamine.

PANC-1 cells. The transfection efficiency is shown in Fig. 5D. Mfn2 overexpression via Ad-Mfn2 blocked the promotive effects of agmir-125a on mitochondrial fission, as demonstrated by less mitochondrial fragmentation or debris in PANC-1 cells (Fig. 5E). These data confirmed the hypothesis that miR-125a triggers mitochondrial fission by inhibiting Mfn2 expression. Additionally, transfection with Ad-Mfn2 also ablated caspase-9 activity, which was upregulated in response to agmir-125a stimulation (Fig. 5F), suggesting that Mfn2 is also associated with mitochondrial fission-mediated mitochondrial apoptosis.

miR-125a is negatively regulated by HIF1. Finally, to elucidate the mechanism by which miR-125a is downregulated in PANC-1 cells, HIF1 was investigated. Several studies have reported that miR-125a is negatively modified by HIF1 and that hypoxic conditions further repress expression of miR-125a $(45,46)$. Thus, it was hypothesized that HIF1 is the upstream regulator of miR-125a. siRNA was used to knockdown expression of HIF1 in PANC-1 cells, and the silencing efficiency of the siRNA is shown in Fig. 6A. Following HIF1 knockdown, mRNA expression of miR-125a was significantly increased. Additionally, hypoxic conditions were induced using a hypoxia chamber with $1 \%$ oxygen to enhance the HIF1 signals. Under hypoxic conditions, miR-125a was further downregulated. Furthermore, DFX was also used to mimic hypoxic conditions, and the results were in agreement with the above findings. These data indicated that miR-125a is negatively regulated by HIF1. To provide further evidence regarding the role of HIF1 in fission, the influence of HIF1 on Mfn2 expression was evaluated. Knockdown of HIF1 also reduced expression of Mfn2, which was increased under hypoxic conditions, suggesting a regulatory role for HIF1 in Mfn2 (Fig. 6B and C). Furthermore, the TEM results indicated that more fragmented mitochondria appeared in response to HIF1 silencing. However, upon exposure to hypoxic conditions, mitochondrial fission was inhibited, as demonstrated by longer mitochondria (Fig. 6D). Collectively, these data confirm that HIF1 inhibits mitochondrial fission via the miR125a/Mfn2 pathways.

\section{Discussion}

The results of the demonstrated that miR-125a targets Mfn2 and inhibits transcription and expression of Mfn2. Furthermore, reduced Mfn2 contributed to excessive mitochondrial fission in PANC-1 cells, which resulted in activation of mitochondria-associated apoptosis pathways. Excessive fission causes cellular energy disorder and migration impairment. Finally, the results of the current study demonstrated that HIF1 is an upstream regulator of miR-125a/Mfn1, and subsequent mitochondrial fission. To the best of our knowledge, this is the first study to describe the tumor-suppressive actions of HIF1/Mfn2/miR-125a in PANC-1 cells, including regulation of cellular apoptosis, energy metabolism and migration by inhibition of mitochondrial fission.

Previous studies have reported that miR-125a has a tumor-suppressive role in various cancer types by negatively regulating tumor oncogenes $(47,48)$. For example, miR-125a is an independent prognostic factor and inhibits the proliferation of gastric cancer (49). Additionally, miR-125a functions as a tumor suppressor by regulating abnormal activity of sirtuin 7 in hepatocellular carcinoma tumorigenesis (50). In the current study, miR-125a enhances the mitochondrial fission that is involved in PANC-1 cell apoptosis, metabolism and migration. At the time of PC diagnosis, the majority of patients are determined to have unresectable disease. Considerable efforts have been made to develop novel therapeutic treatments for this disease; however, progress on this issue is limited. Identifying the appropriate miRNAs and tapping into the excellent potential of miRNAs will have a beneficial impact on the treatment of PC. The present study provides evidence that the concentration of miR-125a is positively associated with PANC-1 cell apoptosis, energy disorders and migration impairment, and may be used as a possible target to regulate PANC-1 cell biological processes. However, more clinical data are required.

Mitochondrial homeostasis is known to be associated with the pathogenesis of neurodegenerative diseases, diabetes and myopathies, among other human diseases. In the present 
study, overexpression of miR-125a induced mitochondrial fission, as demonstrated by increased mitochondrial fragmentation. Furthermore, excessive mitochondrial fission causes mitochondrial depolarization, followed by cyt-c leakage into the cytoplasm (51). As a consequence of cyt-c release, mitochondria-associated apoptosis pathways are activated, as evidenced by increased pro-apoptosis protein expression and decreased anti-apoptosis protein expression (52). Other studies have reported that mitochondrial fission leads to voltage-dependent anion channel 1 oligomerization and the separation of hexokinase 2 (HK2) from the outer mitochondrial membrane, resulting in the opening of the mPTP and reduced mitochondrial membrane potential $(53,54)$. These findings were similar those of the present study, which demonstrated that mitochondrial fission involved in cell apoptosis by damaging the mitochondrial structure and function. Additionally, miR125a-regulated mitochondrial fission is reported to be involved in cellular energy disorder. Excessive mitochondrial fission impaired mitochondrial ATP production by reducing ETCx activity (55), followed by decreased glucose consumption and lactate production. Notably, other studies have indicated that miR-125a has a negative role in the regulation of HK2 (56), a glycolytic rate-limiting enzyme, and therefore has a negative role in the modified Warburg effect in hepatocellular carcinoma. These findings were similar to the data of the present study that demonstrated that miR-125a is the key regulator of cancer cell energy metabolism. Furthermore, in the current study, it was also demonstrated that miR125a-induced mitochondrial fission is involved in PANC-1 cell migration. Increased fragmentation was associated with impaired cellular mobilization (57). As F-actin is the key stress fiber that directly monitors cellular mobilization (58), it was hypothesized that blunted migration arose from mitochondrial fission-involved F-actin dyshomeostasis. The results of the current study indicated that mitochondrial fission leads to F-actin depolymerization to G-actin, eventually causing a considerable obstacle in cellular migration. Successive mitochondrial fission is dependent on binding between Drp1, the Drp1 receptor and stress fibers (59). Previously, studies have indicated that intracellular F-actin accumulation on the surface of mitochondria for short periods of time is a prerequisite for subsequent mitochondrial division $(60,61)$. Under physiological conditions, F-actin is regularly distributed in certain parts of the cytoplasm that control cellular migration to direct cells to move in a particular direction. When mitochondrial fission is initiated, F-actin decomposes into G-actin, which is then reassembled into F-actin at the outer mitochondrial membrane to promote the formation of a contractile ring with the help of Drp1 and its receptor. Considering the indispensable nature of F-actin in fission, excessive fission may consume large amounts of cytoplasmic F-actin, causing an uneven distribution of F-actin (62), ultimately leading to dysregulated F-actin homeostasis and impaired migration. More evidence is required to support this hypothesis. Together, the data provide useful information about the tumor-suppressive role of mitochondrial fission in PANC-1 cells, including apoptosis, migration and energy metabolism. These findings provide a clear target to inhibit PC development and progression.

Dysfunction of mitochondria is largely dependent on Mfn2 activity, which preserves the mitochondrial dynamic balance (fission and fusion) (63). The findings of the current study confirmed that miR-125a can directly target Mfn2 and inhibit Mfn2 transcription and expression. Furthermore, decreased Mfn2 contents were associated with excessive mitochondrial fission. Several studies have illustrated the regulatory role of Mfn2 in mitochondrial fission by counteracting Drp1 $(64,65)$. Furthermore, studies have also reported that Mfn 2 is able to indirectly activate Parkin1-dependent mitophagy, which removes fragmented mitochondria and therefore blocks mitochondrial fission (66). This information describes the comprehensive role of Mfn2 in mitochondrial homeostasis. Several studies have also defined other elements of the mitochondrial fission machinery, including mitochondrial fission factor, Drp1 and Opal mitochondrial dynamin like GTPase $(14,16,67)$. However, whether these factors have anti-tumor properties remains unclear. This may be the mechanism by which Mfn2 regulates mitochondrial fission. Furthermore, the results of the current study demonstrated that miR-125a/Mfn2 is regulated by HIF1, a regulatory protein that was significantly increased under hypoxic conditions. In fact, the normal pancreas has an abundant blood flow, in contrast to pancreatic cancer, which is a hypovascular tumor. This is partly because of fibrotic changes around the tumor due to pancreatitis associated with cancer invasion. In the current study, knockdown of HIF1 enhanced miR-125a expression and promoted mitochondrial fission, suggesting that miR-125a is negatively regulated by HIF1. Additionally, these data also indicated that HIF1 has a tumor-promotive role in PC. In fact, hypoxia and HIF1 are essential components of the neoplastic microenvironment, often allowing a selective advantage for tumor cells over otherwise non-invasive cells that are more sensitive to a low oxygen state. The evidence for hypoxia and HIF expression in pancreatic cancer is in their characteristic avascular appearance on computed tomography and from intratumoral oxygen tension measurements. Hypoxic conditions and HIF1 expression in solid malignancies may confer resistance to conventional radiation and chemotherapy. Therefore, there is a focus on identifying the mechanism by which hypoxia/HIF1 contributes to PC development. The findings of the present study suggest that HIF1 negatively affects miR-125a expression and therefore augments Mfn2 expression, contributing to PANC-1 survival and migration by inhibiting mitochondrial fission.

However, the present study aimed to explore the role of mitochondrial fission in PANC-1 cells rather than in normal pancreatic ductal epithelial cell lines. Thus, additional experimental evidence is required.

In conclusion, the current study described the critical roles of HIF1/miR-125a/Mfn2 in PANC-1 cell apoptosis, migration and energy metabolism via mitochondrial fission. Regulation of mitochondrial fission by HIF1/miR-125a/Mfn1 during PC carcinogenesis may be a potential target to modify tumor growth and promotion, which opens a novel avenue for PC treatment.

\section{Acknowledgements}

Not applicable.

\section{Funding}

No funding was received. 


\section{Availability of data and materials}

The datasets used and/or analyzed during the current study are available from the corresponding author on reasonable request.

\section{Authors' contributions}

LCP, LZ and WJY were involved in conception and design, performance of experiments, data analysis and interpretation, and manuscript writing. LCP, JB, and RL were involved in data analysis and interpretation.

\section{Ethics approval and consent to participate}

Not applicable.

\section{Consent for publication}

Not applicable.

\section{Competing interests}

The authors declare that they have no competing interests.

\section{References}

1. Xu Z, Pothula SP, Wilson JS and Apte MV: Pancreatic cancer and its stroma: A conspiracy theory. World J Gastroenterol 20 11216-11229, 2014.

2. Pinho AV, Chantrill L and Rooman I: Chronic pancreatitis: A path to pancreatic cancer. Cancer Lett 345: 203-209, 2014.

3. Lin QJ, Yang F, Jin C and Fu DL: Current status and progress of pancreatic cancer in China. World J Gastroenterol 21: 7988-8003 2015.

4. He B, Zhao Y, Xu L, Gao L, Su Y, Lin N and Pu J: The nuclear melatonin receptor ROR $\alpha$ is a novel endogenous defender agains myocardial ischemia/reperfusion injury. J Pineal Res 60: 313-326, 2016.

5. Kleszczyński K, Zillikens D and Fischer TW: Melatonin enhances mitochondrial ATP synthesis, reduces reactive oxygen species formation, and mediates translocation of the nuclear erythroid 2-related factor 2 resulting in activation of phase-2 antioxidant enzymes ( $\gamma$-GCS, HO-1, NQO1) in ultraviolet radiationtreated normal human epidermal keratinocytes (NHEK). J Pineal Res 61: 187-197, 2016.

6. Zhang R, Sun Y, Liu Z, Jin W and Sun Y: Effects of melatonin on seedling growth, mineral nutrition, and nitrogen metabolism in cucumber under nitrate stress. J Pineal Res 62: e12403, 2017. doi: 10.1111/jpi.12403.

7. Sandesc M, Dinu A, Rogobete AF, Bedreag OH, Sandesc D, Papurica M, Bratu LM, Negoita S, Vernic C, Popovici SE, et al: Circulating microRNAs expressions as genetic biomarkers in pancreatic cancer patients continuous non-invasive monitoring. Clin Lab 63: 1561-1566, 2017.

8. Liang L, Wei DM, Li JJ, Luo DZ, Chen G, Dang YW and Cai XY: Prognostic microRNAs and their potential molecular mechanism in pancreatic cancer: A study based on The Cancer Genome Atlas and bioinformatics investigation. Mol Med Rep 17: 939-951, 2018.

9. Shi C, Cai Y, Li Y, Li Y, Hu N, Ma S, Hu S, Zhu P, Wang W and Zhou H: Yap promotes hepatocellular carcinoma metastasis and mobilization via governing cofilin/F-actin/lamellipodium axis by regulation of JNK/Bnip3/SERCA/CaMKII pathways. Redox Biol 14: 59-71, 2018

10. Jiang L, Huang Q, Zhang S, Zhang Q, Chang J, Qiu X and Wang E: Hsa-miR-125a-3p and hsa-miR-125a-5p are downregulated in non-small cell lung cancer and have inverse effects on invasion and migration of lung cancer cells. BMC Cancer 10: 318, 2010.

11. Nam EJ, Yoon H, Kim SW, Kim H, Kim YT, Kim JH, Kim JW and Kim S: MicroRNA expression profiles in serous ovarian carcinoma. Clin Cancer Res 14: 2690-2695, 2008.
12. Ferretti E, De Smaele E, Po A, Di Marcotullio L, Tosi E, Espinola MS, Di Rocco C, Riccardi R, Giangaspero F, Farcomeni A, et al: MicroRNA profiling in human medulloblastoma. Int J Cancer 124: 568-577, 2009.

13. Hashiguchi Y, Nishida N, Mimori K, Sudo T, Tanaka F, Shibata K, Ishii $\mathrm{H}$, Mochizuki H, Hase K, Doki Y, et al: Downregulation of miR-125a-3p in human gastric cancer and its clinicopathological significance. Int J Oncol 40: 1477-1482, 2012.

14. Zhou H, Ma Q, Zhu P, Ren J, Reiter RJ and Chen Y: Protective role of melatonin in cardiac ischemia-reperfusion injury: From pathogenesis to targeted therapy. J Pineal Res 64: e12471, 2018. doi: $10.1111 /$ jpi.12471.

15. Das N, Mandala A, Naaz S, Giri S, Jain M, Bandyopadhyay D, Reiter RJ and Roy SS: Melatonin protects against lipid-induced mitochondrial dysfunction in hepatocytes and inhibits stellate cell activation during hepatic fibrosis in mice. J Pineal Res 62: e12404, 2017. doi: 10.1111/jpi.12404.

16. Jin Q, Li R, Hu N, Xin T, Zhu P, Hu S, Ma S, Zhu H, Ren J and Zhou H: DUSP1 alleviates cardiac ischemia/reperfusion injury by suppressing the Mff-required mitochondrial fission and Bnip3-related mitophagy via the JNK pathways. Redox Biol 14: 576-587, 2018.

17. Zhou H, Du W, Li Y, Shi C, Hu N, Ma S, Wang W and Ren J: Effects of melatonin on fatty liver disease: The role of NR4A1/ DNA-PKcs/p53 pathway, mitochondrial fission, and mitophagy. J Pineal Res 64: e12450, 2018. doi: 10.1111/jpi.12450.

18. Zhu H, Jin Q, Li Y, Ma Q, Wang J, Li D, Zhou H and Chen Y: Melatonin protected cardiac microvascular endothelial cells against oxidative stress injury via suppression of IP3R- $\left[\mathrm{Ca}^{2+}\right]$ c/VDAC- $\left[\mathrm{Ca}^{2+}\right] \mathrm{m}$ axis by activation of MAPK/ERK signaling pathway. Cell Stress Chaperones 23: 101-113, 2018.

19. Hu SY, Zhang Y, Zhu PJ, Zhou H and Chen YD: Liraglutide directly protects cardiomyocytes against reperfusion injury possibly via modulation of intracellular calcium homeostasis. J Geriatr Cardiol 14: 57-66, 2017.

20. Zhou H, Zhu P, Guo J, Hu N, Wang S, Li D, Hu S, Ren J, Cao $F$ and Chen Y: Ripk3 induces mitochondrial apoptosis via inhibition of FUNDC1 mitophagy in cardiac IR injury. Redox Biol 13: 498-507, 2017

21. Zhou H, Li D, Zhu P, Hu S, Hu N, Ma S, Zhang Y, Han T, Ren J, $\mathrm{Cao} \mathrm{F}$, et al: Melatonin suppresses platelet activation and function against cardiac ischemia/reperfusion injury via PPARgamma/ FUNDC1/mitophagy pathways. J Pineal Res 63: e12438, 2017. doi: $10.1111 /$ jpi.12438

22. Reiter RJ, Mayo JC, Tan DX, Sainz RM, Alatorre-Jimenez M and Qin L: Melatonin as an antioxidant: Under promises but over delivers. J Pineal Res 61: 253-278, 2016.

23. Fuhrmann DC and Brüne B: Mitochondrial composition and function under the control of hypoxia. Redox Biol 12: 208-215, 2017.

24. Dong X, Fu J, Yin X, Qu C, Yang C, He H and Ni J: Induction of apoptosis in HepaRG cell line by aloe-emodin through generation of reactive oxygen species and the mitochondrial pathway. Cell Physiol Biochem 42: 685-696, 2017.

25. Brasacchio D, Alsop AE, Noori T, Lufti M, Iyer S, Simpson KJ Bird PI, Kluck RM, Johnstone RW and Trapani JA: Epigenetic control of mitochondrial cell death through PACS1-mediated regulation of BAX/BAK oligomerization. Cell Death Differ 24: 961-970, 2017.

26. Gao Y, Xiao X, Zhang C, Yu W, Guo W, Zhang Z, Li Z, Feng X, Hao J, Zhang K, et al: Melatonin synergizes the chemotherapeutic effect of 5-fluorouracil in colon cancer by suppressing PI3K/AKT and NF-kappaB/iNOS signaling pathways. J Pineal Res 62: e12380, 2018. doi: 10.1111/jpi.12380.

27. Dufour F, Rattier T, Shirley S, Picarda G, Constantinescu AA, Morlé A, Zakaria AB, Marcion G, Causse S, Szegezdi E, et al: $\mathrm{N}$-glycosylation of mouse TRAIL-R and human TRAIL-R1 enhances TRAIL-induced death. Cell Death Differ 24: 500-510, 2017.

28. Griffiths HR, Gao D and Pararasa C: Redox regulation in metabolic programming and inflammation. Redox Biol 12: 50-57, 2017.

29. Iggena D, Winter Y and Steiner B: Melatonin restores hippocampal neural precursor cell proliferation and prevents cognitive deficits induced by jet lag simulation in adult mice. J Pineal Res 62: e12397, 2017. doi: 10.1111/jpi.12397.

30. Hambright WS, Fonseca RS, Chen L, Na R and Ran Q: Ablation of ferroptosis regulator glutathione peroxidase 4 in forebrain neurons promotes cognitive impairment and neurodegeneration. Redox Biol 12: 8-17, 2017. 
31. Murphy PS, Wang J, Bhagwat SP, Munger JC, Janssen WJ, Wright TW and Elliott MR: CD73 regulates anti-inflammatory signaling between apoptotic cells and endotoxin-conditioned tissue macrophages. Cell Death Differ 24: 559-570, 2017.

32. Zhou H, Wang J, Zhu P, Hu S and Ren J: Ripk3 regulates cardiac microvascular reperfusion injury: The role of IP3R-dependent calcium overload, XO-mediated oxidative stress and F-action/ filopodia-based cellular migration. Cell Signal 45: 12-22, 2018.

33. Li J, Chen L, Xiong Y, Zheng X, Xie Q, Zhou Q, Shi L, Wu C, Jiang $J$ and Wang H: Knockdown of PD-L1 in human gastric cancer cells inhibits tumor progression and improves the cytotoxic sensitivity to CIK therapy. Cell Physiol Biochem 41: 907-920, 2017

34. Lee $\mathrm{K}$ and Back K: Overexpression of rice serotonin N-acetyltransferase 1 in transgenic rice plants confers resistance to cadmium and senescence and increases grain yield. J Pineal Res 62: e12392, 2017.

35. Jokinen R, Pirnes-Karhu S, Pietiläinen KH and Pirinen E: Adipose tissue $\mathrm{NAD}^{+}$-homeostasis, sirtuins and poly(ADP-ribose) polymerases-important players in mitochondrial metabolism and metabolic health. Redox Biol 12: 246-263, 2017.

36. Yang HH, Chen Y, Gao CY, Cui ZT and Yao JM: Protective Effects of microRNA-126 on human cardiac microvascular endothelial cells against hypoxia/reoxygenation-induced injury and inflammatory response by activating PI3K/Akt/eNOS signaling pathway. Cell Physiol Biochem 42: 506-518, 2017.

37. Liu Z, Gan L, Xu Y, Luo D, Ren Q, Wu S and Sun C: Melatonin alleviates inflammasome-induced pyroptosis through inhibiting NF-kappaB/GSDMD signal in mice adipose tissue. J Pineal Res 63: e12414, 2017. doi: 10.1111/jpi.12414.

38. Lee HJ, Jung YH, Choi GE, Ko SH, Lee SJ, Lee SH and Han HJ BNIP3 induction by hypoxia stimulates FASN-dependent free fatty acid production enhancing therapeutic potential of umbilical cord blood-derived human mesenchymal stem cells. Redox Biol 13: 426-443, 2017.

39. Yu S, Wang X, Geng P, Tang X, Xiang L, Lu X, Li J, Ruan Z, Chen J, $\mathrm{Xie} \mathrm{G}$, et al: Melatonin regulates PARP1 to control the senescenceassociated secretory phenotype (SASP) in human fetal lung fibroblast cells. J Pineal Res 63 e12405, 2017. doi: 10.1111/jpi.12405.

40. Zhang Y, Zhou H, Wu W, Shi C, Hu S, Yin T, Ma Q, Han T, Zhang Y, Tian F, et al: Liraglutide protects cardiac microvascular endothelial cells against hypoxia/reoxygenation injury through the suppression of the SR-Ca(2+)-XO-ROS axis via activation of the GLP-1R/PI3K/Akt/survivin pathways. Free Radic Biol Med 95: 278-292, 2016.

41. Zhou H, Yang J, Xin T, Li D, Guo J, Hu S, Zhou S, Zhang T, Zhang Y, Han T, et al: Exendin-4 protects adipose-derived mesenchymal stem cells from apoptosis induced by hydrogen peroxide through the PI3K/Akt-Sfrp2 pathways. Free Radic Biol Med 77: 363-375, 2014

42. Zhou H, Hu S, Jin Q, Shi C, Zhang Y, Zhu P, Ma Q, Tian F and Chen Y: Mff-dependent mitochondrial fission contributes to the pathogenesis of cardiac microvasculature ischemia/reperfusion injury via induction of mROS-mediated cardiolipin oxidation and HK2/VDAC1 disassociation-involved mPTP opening. J Am Heart Assoc 6: e005328, 2017.

43. Zhou H, Yang J, Xin T, Zhang T, Hu S, Zhou S, Chen G and Chen Y: Exendin- 4 enhances the migration of adipose-derived stem cells to neonatal rat ventricular cardiomyocyte-derived conditioned medium via the phosphoinositide 3-kinase/Akt-stromal cell-derived factor-1 $\alpha / \mathrm{CXC}$ chemokine receptor 4 pathway. Mol Med Rep 11: 4063-4072, 2015.

44. Richard V, Kindt N and Saussez S: Macrophage migration inhibitory factor involvement in breast cancer (Review). Int J Oncol 47: 1627-1633, 2015.

45. Ma C, Zhang C, Ma M, Zhang L, Zhang L, Zhang F, Chen Y Cao F, Li M, Wang G, et al: MiR-125a regulates mitochondrial homeostasis through targeting mitofusin 1 to control hypoxic pulmonary vascular remodeling. J Mol Med (Berl) 95: 977-993, 2017.

46. Van Nostrand JL, Bowen ME, Vogel H, Barna M and Attardi LD: The p53 family members have distinct roles during mammalian embryonic development. Cell Death Differ 24: 575-579, 2017.

47. Zhao JL, Huang F, He F, Gao CC, Liang SQ, Ma PF, Dong GY, Han $\mathrm{H}$ and Qin HY: Forced activation of notch in macrophages represses tumor growth by upregulating miR-125a and disabling tumor-associated macrophages. Cancer Res 76: 1403-1415, 2016.

48. Fang R, Xiao T, Fang Z, Sun Y, Li F, Gao Y, Feng Y, Li L, Wang Y, Liu X, et al: MicroRNA-143 (miR-143) regulates cancer glycolysis via targeting hexokinase 2 gene. J Biol Chem 287: 23227-23235, 2012 .
49. Nishida N, Mimori K, Fabbri M, Yokobori T, Sudo T, Tanaka F, Shibata K, Ishii H, Doki Y and Mori M: MicroRNA-125a-5p is an independent prognostic factor in gastric cancer and inhibits the proliferation of human gastric cancer cells in combination with trastuzumab. Clin Cancer Res 17: 2725-2733, 2011.

50. Kim JK, Noh JH, Jung KH, Eun JW, Bae HJ, Kim MG, Chang YG, Shen Q, Park WS, Lee JY, et al: Sirtuin7 oncogenic potential in human hepatocellular carcinoma and its regulation by the tumor suppressors miR-125a-5p and miR-125b. Hepatology 57: 1055-1067, 2013.

51. Du K, Ramachandran A and Jaeschke H: Oxidative stress during acetaminophen hepatotoxicity: Sources, pathophysiological role and therapeutic potential. Redox Biol 10: 148-156, 2016.

52. Kakimoto PA and Kowaltowski AJ: Effects of high fat diets on rodent liver bioenergetics and oxidative imbalance. Redox Biol 8: 216-225, 2016

53. Xu S, Pi H, Zhang L, Zhang N, Li Y, Zhang H, Tang J, Li H, Feng M, Deng P, et al: Melatonin prevents abnormal mitochondrial dynamics resulting from the neurotoxicity of cadmium by blocking calcium-dependent translocation of Drp1 to the mitochondria. J Pineal Res 60: 291-302, 2016.

54. Zhou H, Zhang Y, Hu S, Shi C, Zhu P, Ma Q, Jin Q, Cao F, Tian F and Chen Y: Melatonin protects cardiac microvasculature against ischemia/reperfusion injury via suppression of mitochondrial fission-VDAC1-HK2-mPTP-mitophagy axis. J Pineal Res 63: e12413, 2017.

55. Perdiz D, Lorin S, Leroy-Gori I and Poüs C: Stress-induced hyperacetylation of microtubule enhances mitochondrial fission and modulates the phosphorylation of Drp1 at616Ser. Cell Signal 39: 32-43, 2017.

56. Jin F, Wang Y, Zhu Y, Li S, Liu Y, Chen C, Wang X, Zen K and Li L: The miR-125a/HK2 axis regulates cancer cell energy metabolism reprogramming in hepatocellular carcinoma. Sci Rep 7: 3089, 2017.

57. Tallman KA, Kim HH, Korade Z, Genaro-Mattos TC, Wages PA, Liu W and Porter NA: Probes for protein adduction in cholesterol biosynthesis disorders: Alkynyl lanosterol as a viable sterol precursor. Redox Biol 12: 182-190, 2017.

58. Zhou H, Wang S, Zhu P, Hu S, Chen Y and Ren J: Empagliflozin rescues diabetic myocardial microvascular injury via AMPKmediated inhibition of mitochondrial fission. Redox Biol 15: 335-346, 2018.

59. Oanh NT, Park YY and Cho H: Mitochondria elongation is mediated through SIRT1-mediated MFN1 stabilization. Cell Signal 38: 67-75, 2017

60. Torres-Quesada O, Mayrhofer JE and Stefan E: The many faces of compartmentalized PKA signalosomes. Cell Signal 37: 1-11, 2017.

61. Schock SN, Chandra NV, Sun Y, Irie T, Kitagawa Y, Gotoh B, Coscoy L and Winoto A: Induction of necroptotic cell death by viral activation of the RIG-I or STING pathway. Cell Death Differ 24: 615-625, 2017.

62. Salminen A, Kaarniranta K and Kauppinen A: Integrated stress response stimulates FGF21 expression: Systemic enhancer of longevity. Cell Signal 40: 10-21, 2017.

63. Park J, Tran Q, Mun K, Masuda K, Kwon SH, Kim SH, Kim DH, Thomas G and Park J: Involvement of S6K1 in mitochondria function and structure in HeLa cells. Cell Signal 28: 1904-1915, 2016.

64. Karbowski M,Lee YJ, Gaume B,Jeong SY, Frank S, Nechushtan A, Santel A, Fuller M, Smith CL and Youle RJ: Spatial and temporal association of Bax with mitochondrial fission sites, Drpl, and Mfn2 during apoptosis. J Cell Biol 159: 931-938, 2002.

65. Song M, Franco A, Fleischer JA, Zhang L and Dorn GW II: Abrogating mitochondrial dynamics in mouse hearts accelerates mitochondrial senescence. Cell Metab 26: 872-883.e5, 2017.

66. Chen Y and Dorn GW II: PINK1-phosphorylated mitofusin 2 is a Parkin receptor for culling damaged mitochondria. Science 340: 471-475, 2013.

67. Banerjee K, Keasey MP, Razskazovskiy V, Visavadiya NP, Jia C and Hagg T: Reduced FAK-STAT3 signaling contributes to ER stress-induced mitochondrial dysfunction and death in endothelial cells. Cell Signal 36: 154-162, 2017.

This work is licensed under a Creative Commons Attribution-NonCommercial-NoDerivatives 4.0 International (CC BY-NC-ND 4.0) License. 